\title{
LA GRAN VELOCIDAD FERROVIARIA EN ESPAÑA: ANCHO DE VÍA Y DUPLO-RED
}

\author{
Luis SANTOS Y GANGES
}

Instituto Universitario de Urbanística. Universidad de Valladolid

\section{GRAN VELOCIDAD FERROVIARIA, CONCEPTO FUNCIONAL Y ALCANCE TERRITORIAL}

La gran velocidad ferroviaria ${ }^{1}$ surgió en Japón y Francia como una respuesta al reto de trasladar numerosos viajeros entre determinadas grandes ciudades mediante un modo veloz y capaz. Pero de la potente resolución de un problema concreto se pasó rápidamente -puesto que ya existían tecnología e industria- a su consideración como oportunidad de desarrollo, es decir, a la demanda de plasmación generalizada en el territorio. Mientras que en Japón la red ordinaria ${ }^{2}$ de ferrocarriles tenía unas características limitadas e incompatibles con las nuevas líneas de gran velocidad para los trenes bala, siendo obligada la consideración de dos redes separadas, en Francia las trazas de nueva construcción para el TGV se integraron en la red estatal.

Qué se entiende por gran velocidad es algo que ha ido evolucionando con las mejoras tecnológicas a lo largo del tiempo, desde velocidades máximas de los trenes de línea en torno a $200 \mathrm{~km} / \mathrm{h}$ en los años setenta y de $250 \mathrm{~km} / \mathrm{h}$ en los ochenta, se ha pasado a los $300 \mathrm{~km} / \mathrm{h}$ en los noventa y a los $350 \mathrm{~km} / \mathrm{h}$ en el presente y el futuro más inmediato. Pero son velocidades alcanzadas en los tramos de mejores características de las líneas nuevas específicamente construidas para ello.

Mas la confusión generalizada que se ha creado en torno al concepto de gran

\footnotetext{
${ }^{1}$ Anotemos que la velocidad es grande o pequeña en castellano, a pesar de que en inglés sea alta o baja, y las expresiones de gran velocidad y pequeña velocidad pertenecen al acervo léxico ferroviario desde el siglo XIX.

${ }^{2}$ Para referirnos a la red férrea existente que no responde a los parámetros de la gran velocidad preferimos usar la adjetivación castellana "ordinaria", en lugar de unos tan habituales como incorrectos barbarismos: el anglicismo "convencional” o el galicismo "clásica".
} 
velocidad no proviene tanto del mencionado progreso tecnológico como sobre todo de la introducción del concepto de red de gran velocidad. La gran velocidad en Europa, fundamentalmente en Francia, Italia, Alemania y Benelux, se ha venido concibiendo como la estructura básica para el transporte de viajeros en grandes relaciones, perfectamente integrada en la red general: los trenes de gran velocidad son capaces de discurrir por todos los tipos de vías electrificadas, eso sí, con el mismo ancho de vía. Pero el atractivo político y la demanda de este tipo de inversión pública, y fundamentalmente las propuestas paneuropeas de una red de gran velocidad, vinieron a introducir cierta complejidad. Porque la red de gran velocidad no la forman sólo las nuevas líneas de gran velocidad, sino que se introducen además las líneas ordinarias convenientemente mejoradas para discurrir a velocidades apreciables y aquellas líneas imprescindibles para el efecto red a pesar de no ser ni del primer ni del segundo tipo.

Desde 1984 (pocos años después de entrar en servicio la nueva línea ParísLyón), la Comisión Europea presentó diversas propuestas acerca del enlace de las grandes ciudades europeas mediante líneas ferroviarias de gran velocidad y líneas específicamente acondicionadas. Y ya en 1986 propuso al Consejo de Ministros de Transportes la creación de una red comunitaria, contando siempre con el apoyo del Parlamento Europeo. El esquema director de la "red europea de alta velocidad", propuesto en 1990 y posteriormente definido por un Grupo de Trabajo de Alto Nivel, tipificó las líneas férreas de interés comunitario según las velocidades-tipo: líneas nuevas de alta velocidad, líneas adaptadas a 200 $\mathrm{km} / \mathrm{h}$, líneas mejoradas a $160 \mathrm{~km} / \mathrm{h}$ y líneas de conexión y prolongación. Éste fue el origen de la confusión conceptual, que en España se tradujo con las desafortunadas expresiones de "alta velocidad" y "velocidad alta".

El diferente sentido del concepto "alta velocidad" aplicado a las líneas y a las redes es algo legalmente resuelto en el Real Decreto 1191/2000, de 23 de junio, sobre interoperabilidad del sistema ferroviario de alta velocidad. En su Anexo I "Sistema ferroviario transeuropeo de alta velocidad", punto 1 "Infraestructuras", se dicta que las líneas de alta velocidad incluirán tres tipos de infraestructuras: las especialmente construidas para la alta velocidad (equipadas para velocidades por lo general iguales o superiores a $250 \mathrm{~km} / \mathrm{h}$ ), las especialmente acondicionadas para la alta velocidad (equipadas para velocidades del orden de $200 \mathrm{~km} / \mathrm{h}$ ) y las especialmente acondicionadas para la alta velocidad de carácter específico debido a dificultades topográficas o de entorno urbano (cuya velocidad deberá ajustarse caso por caso). Estas líneas acondicionadas de carácter es- 
pecífico son también llamadas líneas de mallado y líneas de enlace, en particular las conexiones de líneas nuevas o acondicionadas para alta velocidad con las estaciones del centro de las ciudades. Por lo tanto, el asunto queda zanjado definitivamente al asumirse las nociones de la red transeuropea de ferrocarriles de alta velocidad de los años noventa: líneas nuevas, líneas acondicionadas y líneas de enlace.

\subsection{Variaciones en la movilidad y efectos socio-económicos estructurales.}

La competitividad del sistema de gran velocidad es enorme frente a todos los modos de transporte, modificando radicalmente la estructura modal, a lo que debe añadirse un importante efecto clientela, es decir, la movilidad inducida u originada por el propio servicio. Se trata, en su mayor parte, de unos movimientos relacionados con el trabajo y el ocio. Las facilidades para hacer un viaje rápido y cómodo inducen a las empresas a realizar operaciones cara a cara en vez de servirse de las telecomunicaciones, permiten hacer viajes de ida y vuelta en el mismo día y favorecen la propia decisión de desplazarse. Algo que se ha comprobado en todos los casos existentes en el mundo, también en la línea Madrid-Sevilla.

Buena parte de la justificación de la implantación de la gran velocidad descansa en supuestos beneficios de desarrollo regional. Los beneficios observados en el sector servicios para el TGV-Sudeste no parece que puedan ser considerados como argumento de peso en las decisiones de invertir en gran velocidad (NASH, 1991), aunque el $\mathrm{AVE}^{3}$ Madrid-Sevilla tiende a ser presentado como caso exitoso, sobre todo Ciudad Real. Ciertamente, el efecto dinamizador de la gran velocidad desborda la estricta función de transportar (HOUÉE, 1992). El Tren de Gran Velocidad o TGV es también un argumento publicitario, un elemento que aporta calidad y tiene tirón en la imagen de las empresas y de las ciudades (grandes proyectos urbanos). Por ello, se podría pensar que el ferrocarril de gran velocidad influye en las actividades turísticas, en el sector inmobiliario e incluso en la localización de empresas. Según la experiencia francesa y también por los estudios realizados en España (MOPTMA, 1993), podría afirmarse que se trata de un efecto difuso pero incontrovertible sobre la dinamización económica.

\footnotetext{
${ }^{3}$ AVE significa Alta Velocidad Española, y es tanto la marca comercial del servicio como el apelativo del tren eléctrico autopropulsado de viajeros que lo cubre y el nombre
} 
Entre los factores que inciden en las nuevas localizaciones empresariales, parece ser que la evolución del sector tiene mucho más peso que la de los medios de transporte siempre que la accesibilidad sea relativamente buena. En cuanto al desarrollo de las actividades turísticas, el TGV parece ser que ha reactivado o ha consolidado el turismo en aquellos sitios con atractivo reputado. Además, forma parte de numerosos paquetes turísticos, lo que está ocurriendo también en España, vinculado al turismo de congresos y reuniones, que precisa de relaciones rápidas como las del TGV. En general se considera que la mejora sensible de la accesibilidad es una potencialidad para el desarrollo de las actividades turísticas. Por último, las modificaciones urbanísticas tienen consecuencias más tenues de lo que se pensaba. Se crean lugares de actividad económica junto a las estaciones, acondicionados a menudo para usos terciarios e industriales avanzados, pero se registra una lentitud inesperada en alcanzar el pleno funcionamiento. El mercado inmobiliario sí parece que se ve enormemente modificado, siendo el dinamismo constructivo y los aumentos de los precios del suelo los cambios más relevantes.

\subsection{Los posibles efectos territoriales}

La experiencia demuestra que los proyectos de alta velocidad se plantean siempre en los ejes más consolidados de transporte, ya sea para resolver problemas de capacidad de los medios existentes (corredores congestionados y cuellos de botella), ya sea para atajar el problema de las grandes demandas de tráfico, ya sea para recuperar la demanda ferroviaria reduciendo los costes temporales del transporte. Aunque también se ha presentado como oportunidad estratégica de desarrollo regional y como factor de competitividad urbana.

Las líneas de alta velocidad pueden tener la virtud, como cualquier línea nueva, de reactivar el transporte ferroviario, pero por su naturaleza de gran y novedosa actuación es muy difícil inferir sus efectos a largo plazo. Además, los efectos territoriales y socioeconómicos de la implantación y funcionamiento de estas líneas son aún poco conocidos, existiendo hipótesis muy diversas.

Debe ser planteada la cuestión de si la gran velocidad es el instrumento para una nueva organización del territorio. Todos asumen que es un elemento organizador del espacio geográfico, pero el alcance y la valoración de los efectos

del sector del negocio de Renfe.

Polígonos. Revista de Geografía, 11-12 (2001-2002); pp. 137-165. 
son asuntos polémicos y poco estudiados. Por un lado, es indudable que se producen cambios importantes en las accesibilidades relativas: la disposición de la nueva línea puede seguir o no un eje de desarrollo regional, o puede optar por un nuevo itinerario con distintas estaciones de paso.

El nuevo sistema acerca a los principales centros económicos entre sí, es un factor de desenclavamiento allí donde hay estación (ARDUIN, 1991). Dado que la accesibilidad es una condición para el desarrollo, las ciudades comunicadas por la gran velocidad tienen una ventaja competitiva. Los puntos de parada de la nueva línea pueden transformar el dinamismo de las ciudades seleccionadas. Por el contrario, quedar al margen de una línea de gran velocidad puede tener diversos efectos negativos con eventuales repercusiones en el dinamismo de las ciudades, modificando incluso la jerarquía urbana. Frente al riesgo de marginación, otro riesgo menos valorado pero no menos relevante es el de satelización por parte de las grandes ciudades hacia las medias y pequeñas cada vez más cercanas.

Los cambios en la malla ferroviaria son evidentes; los itinerarios del ferrocarril ordinario o convencional ven trocado su papel en el sistema de transporte dado que la gran velocidad canaliza los flujos por otro itinerario. Diversos servicios regionales quedan desfavorecidos porque no pueden aprovechar la línea y porque pierden parte de los viajeros por su causa, mientras que los servicios de mercancías pueden tener mayor disponibilidad de las vías para su desarrollo.

Pero la clave de las posibles nuevas características espaciales inducidas por una futura red de gran velocidad reside en la concepción del territorio como el espacio explotado por la red de grandes ciudades. En este sentido, la gran velocidad es un potente instrumento con eventuales efectos desequilibrantes (PLASSARD, 1991). La gran velocidad puede suponer un efecto de contraste; el acercamiento de una ciudad a una gran metrópoli beneficiaría en principio a la urbe más desarrollada. No se ha cumplido en la experiencia del TGV-Sudeste ninguno de los discursos contrapuestos en este sentido: ni el optimista (efecto de difusión automática de actividad) ni el pesimista (efecto polarizador, tal como ocurrió en Tokio-Osaka).

Estas grandes inversiones suelen hacerse allí donde el efecto de masificación es mayor, en los grandes ejes de transporte. Este efecto de concentración es menos desequilibrador si la red de gran velocidad es compatible con la ordinaria, tal como ocurre en Francia, Alemania o Italia. En España no es así debido al di- 
ferente ancho de vía utilizado (ancho internacional para las líneas de gran velocidad y ancho ibérico para las líneas ordinarias de Renfe).

La gran velocidad surgió para conectar grandes ciudades (Tokio-Osaka, ParísLyón) evitando el paso por las ciudades intermedias, de forma que el territorio resultaba más polarizado, favoreciendo claramente a las metrópolis, que son centros de atracción de los nodos logísticos. Sería de este modo un espacio más discontinuo y dual; el resultado del efecto túnel, que impacta e ignora el medio que atraviesa. Dicho de otra forma -más radical pero no menos cierta-, la gran velocidad concebida como modo super-especializado no conferiría accesibilidad al territorio, sino a las grandes urbes. Por el contrario, si se pretenden efectos estructurantes del territorio y si el objetivo principal ya no es tanto resolver la saturación del sistema de transporte o satisfacer una gran demanda entre centros urbanos, sino potenciar el sistema de ciudades a la vez de que mejoran los tiempos de recorrido, la gran velocidad sería un instrumento del desarrollo regional.

\section{2.a. Comunicar grandes ciudades apoyando al sistema urbano}

La red europea de ferrocarriles de gran velocidad propuesta por la Comisión Europea, por su carácter selectivo y especializado, ha de tener por nodos las grandes capitales europeas. Ahora bien, aun aceptando como inevitable que las mayores metrópolis sean las más beneficiadas por el nuevo sistema, hay formas bien distintas de conformar la nueva red de alta velocidad, en función del peso que se dé al criterio de pasar por las ciudades intermedias.

En Francia no son escasos los ejemplos de nuevas líneas que evitan el paso por las ciudades intermedias, siendo muy significativas la línea París-Lyón, que no discurre por Dijón, y la París-Lille, que no lo hace por Nantes. Aun estando conectada con la red ordinaria, los engarces se plantean fundamentalmente en las periferias a modo de antenas para aprovechar mejor el mercado potencial de una nueva red radiocéntrica al mismo tiempo superpuesta al ferrocarril convencional y relativamente aislada para gestionar mejor su especialización. Por lo tanto, la gran velocidad condiciona al sistema urbano francés, modificando su estructura general mediante el olvido o el apoyo a determinadas urbes. En Italia o en Alemania, sin embargo, la gran velocidad se conforma como la estructura troncal de la malla férrea, interconectada perfectamente con la red ordinaria, de manera que no tiende a modificar al sistema de asentamientos urbanos. Algo que, con matices, sucede también en el caso español, sólo distinto en tanto en 
cuanto las ciudades están más dispersas por el territorio y existe el problema del ancho de vía.

Así, en España la gran velocidad se está implantando sobre la base de un criterio territorial básico: pasar por las ciudades existentes aún a costa de cierto rodeo o de mayor coste de construcción. Se está formando una nueva red radial cuyos nodos son las capitales de provincia ${ }^{4}$, de tal suerte que cada línea de gran velocidad se plasma como un rosario donde las cuentas son las ciudades existentes. Se minimiza así el efecto túnel de una infraestructura que nació para unir grandes ciudades.

\section{2.b. La necesidad de renovar el ferrocarril ordinario}

La mayor parte de las valoraciones sobre el sistema de gran velocidad ferroviaria insisten en su carácter rupturista, en la oportunidad estratégica para el sistema de transportes. Sin embargo, implantar un sistema de gran velocidad no es la única política posible (NEBOT, 1992 y ESTEVAN \& SANZ ALDUÁN, 1996). La gran velocidad, en cualquier caso, tiene evidentes virtudes y campos de actuación idóneos en el sector del transporte, pero también tiene claras limitaciones: necesidad de grandes frecuencias y gran número de viajeros, escasa competitividad por debajo de 1 hora y por encima de 3 horas de trayecto, fronteras técnicas para las relaciones internacionales, elevados costes de construcción, rentabilidad económica a menudo dudosa, enorme consumo de energía, mayor impacto ambiental, etc.

Las líneas de gran velocidad, además, no supondrán nunca por sí solas la mejora del ferrocarril y del transporte terrestre, a pesar de que se defienda lo contrario desde algunos sectores de opinión. La modernización de la red debe basarse indefectiblemente en una intensa mejora del ferrocarril ordinario, conjugando unas aceptables velocidades máximas con la elevación de las velocidades mínimas de circulación y conectando centros de actividad con puertos, aeropuertos y plataformas logísticas. El transporte ferroviario de mercancías precisa de una gran atención, aunque a menudo se ve soslayado frente al atractivo de los TGV; la modernización de los ferrocarriles exige actuar tanto para los viaje-

\footnotetext{
${ }^{4}$ Llama la atención el criterio radiocéntrico arborescente de la futura red, que en su esfuerzo por alcanzar las fronteras y las mayores ciudades portuarias dispone todas sus ramas para conectar la capital del reino con las capitales provinciales; una visión bastante parecida a la que se plasmó en la legislación y en las políticas de ferrocarriles de la
} 
ros como para las mercancías. Y esto es así dado que la gran velocidad se especializa en viajeros y en largos recorridos (ampliable a itinerarios regionales y a mercancías de escaso peso) pero deja fuera fundamentalmente a las cercanías y al transporte de mercancías. Pero son estos tipos de transporte los que más tienen que ver con la eficiencia urbana, la competitividad industrial o la congestión de las carreteras, hoy por hoy el sistema universal de transporte.

Por otro lado, incluso si se tiene en cuenta tan sólo el tiempo de transporte como elemento de medición de la modernización del ferrocarril, es decir, considerando la necesidad del ahorro de tiempo de recorrido en un itinerario completo, la actuación puede hacerse o bien eliminando las limitaciones de velocidad o bien actuando en los tramos más rápidos. Para entender esto, téngase en cuenta que pasar de 60 a $65 \mathrm{~km} / \mathrm{h}$ tiene el mismo resultado que pasar de 160 a 200 km/h: 4'5 segundos de ganancia en cada kilómetro (NEBOT, 1991). Por ello, para que el ferrocarril sea más rápido hay que actuar sobre los tramos más lentos: en una red con frecuentes tramos lentos, o limitaciones parciales de velocidad, tiene una enorme importancia actuar en ellos. Su relación con estructuras o con difícil orografía no quiere decir que la actuación sea relativamente más cara; téngase presente que en los tramos rápidos se exige una fuerte inversión puesto que es preciso incrementar mucho más la velocidad para obtener el mismo resultado en tiempo. Existen multitud de matizaciones a lo expuesto, pero en todo caso está claro que no se puede afirmar a priori que la actuación en los tramos rápidos sea más económica.

Por último, debe anotarse que existe material móvil idóneo para la circulación en trazados sinuosos, como el Talgo pendular $\mathrm{u}$ otros trenes con sistemas de basculación, que suele presentar unas velocidades comerciales muy superiores a las del material convencional en los itinerarios de traza más difícil. La modernización de las líneas férreas ya existentes y la explotación de nuevas tecnologías en el material rodante es una solución que no debe ser desdeñada. Es la opción adoptada en países tan diversos como Suecia, Gran Bretaña o Suiza.

\section{UN RECORRIDO POR LA PLANIFICACIÓN FERROVIARIA, 1987-2003.}

La política ferroviaria española en la etapa democrática iniciada en los años

segunda mitad del siglo XIX.

Polígonos. Revista de Geografía, 11-12 (2001-2002); pp. 137-165. 
setenta del siglo XX ha tenido notorios altibajos, por el déficit planificador, por la escasa capacidad inversora o por la imposición de decisiones puntuales. Asuntos de tanta trascendencia como el papel del modo ferroviario en el transporte, la estructura de la red, las actuaciones infraestructurales de primer orden, el ancho de vía o la implantación del sistema de gran velocidad ferroviaria, han sido planteados de modo diverso, incluso contradictorio, en las últimas décadas. Sin embargo, muy pronto se sentaron las bases generales del tratamiento del medio ferroviario, con cuatro objetivos que se han mantenido firmes desde 1984 hasta la actualidad: especializar el transporte ferroviario, aceptar el papel secundario del ferrocarril, aclarar el papel del Estado en Renfe y sobreponer los factores económicos a los tecnológicos. Y desde los años noventa se han sumado otras dos estrategias de actuación: liberalización del sector ferroviario en el marco de la integración europea y concentración de las inversiones en la gran velocidad. En concreto, los hitos en la política ferroviaria reciente pueden ser resumidos del siguiente modo:

1981 Plan General de Ferrocarriles (PGF), sin aprobar por el Gobierno de la Nación.

1984 Acuerdo del Consejo de Ministros para la Suspensión del tráfico en las líneas altamente deficitarias desde el 1-I-85.

Informe de la Comisión para el estudio de los ferrocarriles españoles.

1987 Plan de Transporte Ferroviario 1987-2000 (PTF).

Ley 16/1987 de 30 de julio, de Ordenación de los Transportes Terrestres (LOTT).

1988 Acuerdo del Consejo de Ministros del 9-XII-88 sobre ferrocarriles de alta velocidad y ancho internacional.

1990 Modelo de Gestión de Renfe basado en Unidades de Negocio.

Real Decreto 1211/1990 de 28 de septiembre, Reglamento de la LOTT (ROTT).

1991 Directiva 91/440/CEE sobre desarrollo de los ferrocarriles comunitarios.

1992 Inauguración de la línea y los trenes de gran velocidad Madrid-Sevilla (AVE).

1993 Decisión del Consejo 93/628/CEE relativa a la creación de una red transeuropea de transporte combinado.

Plan Director de Infraestructuras 1993-2007 (PDI).

1994 Real Decreto 121/1994, de 28 de enero, por el que se aprueba el nuevo Estatuto de Renfe. 
Aprobación de la lista de proyectos prioritarios de las redes de transporte transeuropeas en el Consejo de Essen.

1995 Directiva 95/18/CE sobre concesión de licencias a las empresas ferroviarias. Directiva 95/19/CE sobre la adjudicación de las capacidades de la infraestructura ferroviaria y la fijación de los correspondientes cánones de utilización.

Resolución del Consejo, de 19 de junio, sobre el desarrollo del transporte feroviario y del transporte combinado.

1996 Libro Blanco "Estrategia para la revitalización de los Ferrocarriles Comunitarios" Com. (96) 421 final (1996).

Plan de Infraestructuras Ferroviarias 1995-2000 (PIF) que sólo llegó a ser aprobado técnicamente.

Directiva 96/48/CE del Consejo, de 23 de julio, relativa a la interoperabilidad del sistema ferroviario transeuropeo de alta velocidad.

Artículos 160 y 161 de la Ley 13/1996, de 30 de diciembre, que crean el Ente público Gestor de Infraestructuras Ferroviarias (GIF).

1997 Real Decreto 613/1997, de 25 de abril, aprobatorio del Estatuto del GIF. Artículo 104 de la Ley 66/1997 de 30 de diciembre, sobre la normativa de derechos de acceso y tránsito por las infraestructuras ferroviarias españolas de las agrupaciones internacionales de empresas ferroviarias establecidas en la Unión Europea.

1998 Real Decreto 2111/1998 de 2 de octubre, por el que se regula el acceso a las infraestructuras ferroviarias.

2000 Real Decreto 1191/2000 sobre la interoperabilidad del sistema ferroviario. Plan de Infraestructuras de Transporte 2000-2007 (PIT), que incluye un Programa de Infraestructuras Ferroviarias.

2001 Directiva 2001/12/CE por la que se modifica la Directiva 91/440/CEE sobre el desarrollo de los ferrocarriles comunitarios.

Directiva 2001/13/CE por la que se modifica la Directiva 95/18/CE sobre concesión de licencias a las empresas ferroviarias.

Directiva 2001/14/CE relativa a la adjudicación de la capacidad de infraestructura ferroviaria, aplicación de cánones por su utilización y certificación de la seguridad, que deroga la directiva 95/19/CE.

Directiva 2001/16/CE relativa a la interoperabilidad del sistema ferroviario transeuropeo de ferrocarril convencional.

Libro Blanco "La política europea de transportes de cara al 2010: la hora de elegir". 
2003 Ley 39/2003, de 17 de noviembre, del Sector Ferroviario, que crea el ADIF Ente Público Administrador de Infraestructuras Ferroviarias- y RENFE Operadora.

\subsection{Del PTF de 1987 al PIF de 1995: la introducción del AVE}

Tras muchos años sin planificación ferroviaria a largo plazo (el Plan General de Ferrocarriles de 1981 sólo fue aprobado por el Consejo de Administración de Renfe) y superando un largo periodo de crisis económica, salió adelante el Plan de Transporte Ferroviario 1987-2000 (PTF), que fue un intento notable de modernización del ferrocarril de Renfe, volcándose en las mercancías, las grandes relaciones y las cercanías, y actuando fundamentalmente en la infraestructura para conseguir una red básica sin estrangulamientos, mediante la construcción de nuevos grandes accesos y la concentración de la inversión en los corredores de mayor demanda.

Con posterioridad al PTF, la paulatina incorporación de criterios nuevos en la política gubernamental, como el concepto de la gran velocidad ferroviaria o como la mentalidad "europeísta" acerca de la conveniencia de cambiar el ancho de vía ibérico al ancho internacional, fraguaron en el Acuerdo del Consejo de Ministros del día 9 de diciembre de 1988, que dejó viejo al PTF cuando apenas había comenzado a desarrollarse.

La Administración, sin embargo, hablaba del PTF ampliado, cuyas nuevas orientaciones -las del acuerdo gubernamental- eran la construcción de líneas de gran velocidad (prioritariamente Madrid-Sevilla y Madrid-Barcelona-Frontera francesa), la inclusión de la perspectiva europea en la configuración de la red férrea, la introducción del ancho de vía internacional para las nuevas líneas y el planteamiento técnico de la cuestión del cambio del ancho de vía para el ferrocarril de Renfe. Desde entonces, cuantiosas inversiones se dirigieron a la implantación del sistema de gran velocidad ferroviaria en la relación MadridSevilla, mientras el mantenimiento y la mejora infraestructural de las redes ordinarias experimentaron cuando menos una ralentización y una desigual distribución.

Ya en 1993, cuando se presentó el Plan Director de Infraestructuras (PDI), la política ferroviaria estaba enmarcada por el PTF ampliado, el éxito comercial de la línea Madrid-Sevilla (el AVE: Alta Velocidad Española) inaugurada un año antes y los diversos esquemas de una futura red europea de ferrocarriles de gran velocidad (propuestas de la Unión Internacional de Ferrocarriles y de la Comi- 
sión Europea).

El PDI, el primer plan plurimodal de infraestructuras en España, era un sólido documento oficialmente aprobado y ampliamente debatido entre 1993 y 1994. El PDI señalaba como criterio básico que el ferrocarril necesitaba transformarse en un modo especializado "dirigido a los segmentos del mercado en los que presenta evidentes ventajas comparativas respecto a los otros modos". El 28\% del esfuerzo inversor para transporte interurbano correspondía al ferrocarril. Y un tercio de la financiación sería extrapresupuestaria (fondos europeos y privados), orientada al ferrocarril de gran velocidad fundamentalmente. Las propuestas pretendían contribuir a la especialización del modo ferroviario "consolidando una red moderna que le permita la prestación de servicios competitivos con otros modos". Para aplicar esta estrategia se desarrollaron diversos tipos de actuaciones programadas: alta velocidad, actuaciones estructurantes, actuaciones complementarias, seguridad, conservación y mantenimiento, cercanías y redes arteriales ferroviarias.

El PDI configuraba una red ferroviaria básica insertada en la red europea y articulada por las líneas de gran velocidad, que recogían el planteamiento de la red de interés comunitario. Pero se trataba realmente de dos redes ferroviarias diferenciadas: la red de alta velocidad en ancho internacional y la red convencional en ancho ibérico, "coordinadas aprovechando mutuamente sus potenciales respectivos" (MOPTMA, 1994, 148). El PDI formaba, así, una mini-red de gran velocidad y actuaba sobre la red ordinaria mediante una batería de actuaciones que incluía "antenas de la alta velocidad ancho Renfe", "adecuación de líneas a velocidad alta y ancho Renfe", "modernización de líneas y adecuación de la velocidad" y "actuaciones complementarias". La modernización del ferrocarril comenzó entonces a ser considerada como una necesidad social, suponiendo el documento del plan una interesante base para el debate.

Como desarrollo de la estrategia del PDI, entre 1994 y 1995 se preparó una serie de documentos técnicos que habrían de servir de base para la realización de un Plan de Infraestructuras Ferroviarias (PIF). El cambio de gobierno de 1996 lo paralizó sine die hasta que se anunció el Plan de Infraestructuras 20002007.

\subsection{El PIT 2000-2007: hacia la red de alta velocidad}

El Plan de Infraestructuras del Transporte 2000-2007 (PIT) es un material do- 
cumental del Ministerio de Fomento, tan anunciado y aparentemente ya definido como poco conocido 5 . Si bien es cierto que el Ministerio de Fomento en el verano de 2000 anunció ante los medios de comunicación el Plan de Infraestructuras 2000-2007 y el subsiguiente Programa de Infraestructuras Ferroviarias, en la actualidad aún no han sido aprobados ni por el Gobierno de la Nación ni por el Parlamento. Por consiguiente, se trata de una planificación sui generis, si es que merece tal denominación.

Pero lo que sí parece claro es que la inversión presupuestada para infraestructuras ferroviarias experimenta un aumento sin paliativos. Ahora bien, las actuaciones en gran velocidad ferroviaria suponen una partida veinte veces superior a la dedicada a las cercanías y a la modernización de la red ordinaria juntas. Es decir, el PIT de 2000 conforma -al igual que el PDI de 1993- dos redes ferroviarias, pero con la diferencia de que la mini-red de gran velocidad y ancho internacional se convierte en una red generalizada por el territorio, mientras que la red ordinaria pasa a un último plano, es un resto. La lógica funcional de la gran velocidad ferroviaria en ancho internacional ha perdido las restricciones, el pudor y las contradicciones anteriores y se ha desarrollado plenamente, aunque a costa de obviar al ferrocarril preexistente.

Hasta ahora, la gran velocidad ferroviaria está funcionando como tal en una sola línea al tiempo que se están construyendo otras nuevas. Pero no ha existido el planteamiento de una auténtica red, sino que estas líneas junto con otras a mejorar formaban el armazón de la red, la red ferroviaria básica. Aunque era en parte una entelequia presentar las líneas de ancho internacional como vertebra-

\footnotetext{
${ }^{5}$ Conocemos tan sólo una pequeña parte de la documentación del publicitado plan por las siguientes referencias: el anuncio del presidente del Gobierno ante el Parlamento el 25 de abril de 2000 (intervención en el Debate de Investidura); las intervenciones del ministro de Fomento ante la Comisión de Infraestructuras del Congreso de los Diputados el 15 de junio de 2000 y ante la del Senado el 21 de septiembre de 2000; las jornadas "Viaria" realizadas en Galicia, Extremadura y Castilla y León; un documento de presentación del plan entregado a los diputados del Congreso -en el que el Programa de Infraestructuras Ferroviarias abultaba nada menos que dos páginas de texto y dos mapas esquemáticos-, por las notas de prensa oficiales "El Gobierno informa", y sobre todo por el material divulgativo de la página web ministerial (www.mfom.es), donde está colgado desde el año 2001 el mapa de la futura red de alta velocidad, y, desde noviembre de 2003, el documento del PIT, de un centenar de páginas (que sólo dedica a ferrocarriles las págs. 13-16 de sumaria explicación y las págs. 69-85 de memoria del cumplimiento de lo previsto).
} 
doras del conjunto de la red, dado su distinto ancho de vía, el PDI establecía que éstas siempre debían basar su "complementariedad con la malla convencional" (MOPTMA, 1994, 148). El PIT 2000-2007, sin embargo, dispone la creación de una "red de alta velocidad", es decir, una serie de líneas de altas prestaciones independiente en lo posible de las líneas ordinarias.

El Ministerio de Fomento plantea, en el marco del Programa de Infraestructuras Ferroviarias, la construcción de una auténtica red de líneas de gran velocidad en el horizonte 2007-2010, si bien sin distinguir nunca el tipo de infraestructura de cada línea: especialmente construida para la alta velocidad (línea nueva), especialmente acondicionada para la alta velocidad (línea acondicionada) o especialmente acondicionada para la alta velocidad de carácter específico debido a dificultades topográficas o de entorno urbano (línea de enlace). Debido a esto, la confusión o el equívoco es patente, máxime cuando el propio Ministerio elabora proyectos con una denominación aparente de acondicionamiento de líneas para alta velocidad, como es el caso del Estudio Informativo de la Mejora del Ferrocarril de Medina del Campo a Puebla de Sanabria, cuando resulta que la opción elegida es una línea nueva especialmente construida para la alta velocidad con tramos especialmente acondicionados con carácter específico, que no son sino los enlaces a la ciudad de Zamora.

Debe tenerse en cuenta que las líneas especialmente acondicionadas para alta velocidad a menudo construyen variantes locales, es decir, tramos nuevos que evitan rodeos, curvas cerradas y constreñimientos; es decir, en un trayecto determinado pueden coincidir tramos especialmente construidos con los tramos especialmente acondicionados. Esta complejidad hace a veces difícil adjudicar un carácter concreto a los tramos férreos de la red de alta velocidad, pero no es justificación suficiente para plasmar en el mapa un esfuerzo de mínimos en la distinción entre las categorías establecidas. No se trata sólo de que el carácter flexible del plan le permite evolucionar o incorporar nuevas propuestas (por ejemplo, acceso de Ávila a la red de alta velocidad por Segovia, o el denominado "Plan Galicia", que incorpora dos nuevos corredores, denominados cantábrico y subcantábrico, que supondrán más de 650 nuevos kilómetros de línea), sino de que es un material inacabado y sujeto a malentendidos por su propia indefinición. Así, el reciente acuerdo de los gobiernos español y portugués que establece una estrategia básica común en la formación futura de una red ibérica de líneas de gran velocidad, aún no ha sido incorporado al PIT.

La red de gran velocidad prevista en octubre de 2003 constaría de unos 7.700

Polígonos. Revista de Geografía, 11-12 (2001-2002); pp. 137-165. 
kilómetros de línea, es decir, una ambiciosa red ferroviaria en un plazo relativamente corto. Así lo entiende incluso la Unión Internacional de Ferrocarriles, cuya estimación para el año 2010 reduce su longitud probable a unos $2.600 \mathrm{~km}$, toda vez que a mediados de 2003 están en obras unos $1.500 \mathrm{~km}$. Una nueva red que, salvo por el corredor mediterráneo, será notoriamente radiocéntrica; de hecho, el propio Ministerio de Fomento utiliza frecuentemente el argumento del tiempo de transporte inferior a las tres horas en las relaciones entre Madrid y cualquier capital de provincia.

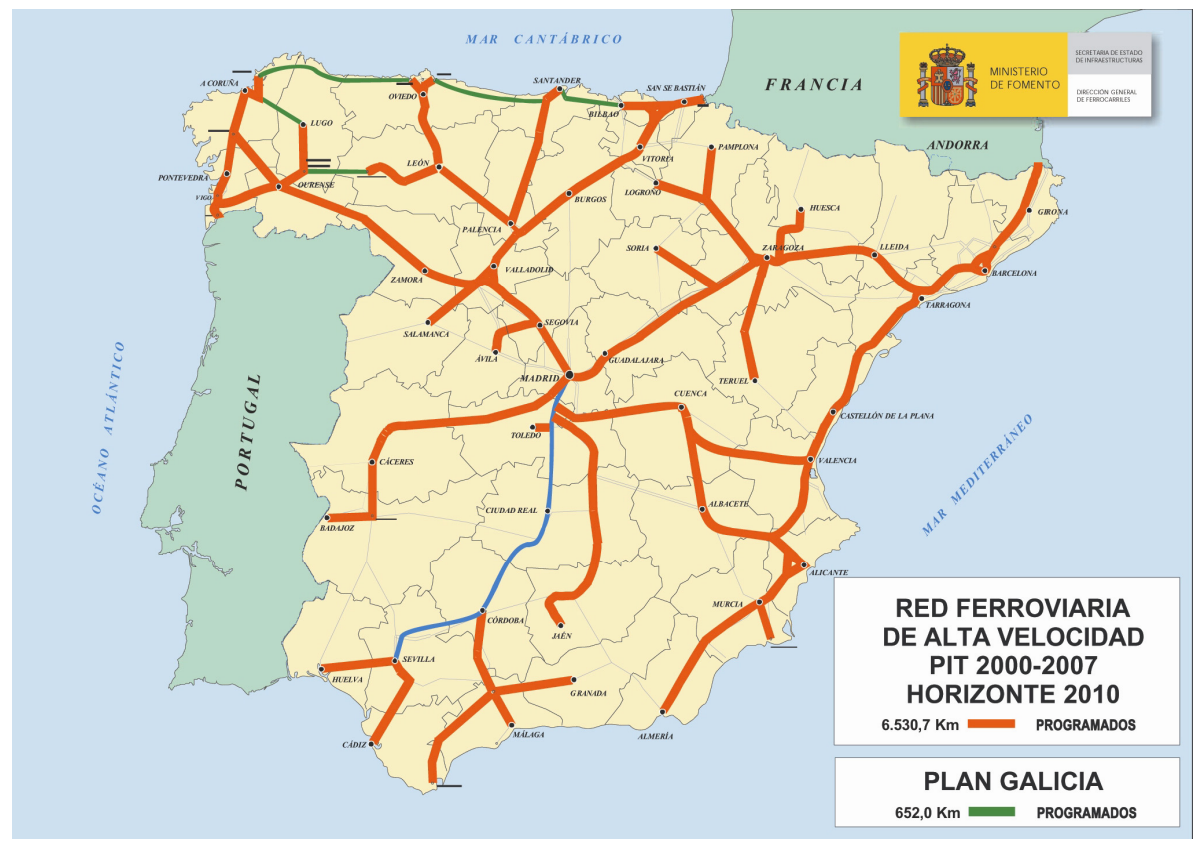

FUENTE: www.mfom.es, a noviembre de 2003.

El PIT, por otro lado, crea la red de alta velocidad ferroviaria insertándola en la correspondiente red transeuropea, en general aplicando las especificaciones técnicas de interoperabilidad emanadas de la directiva europea que se ha visto transpuesta en el correspondiente Real Decreto. 


\subsection{Las redes transeuropeas en las que interviene el ferrocarril}

La Comisión Europea viene elaborando, desde hace más de quince años, sucesivas propuestas de esquemas directores de las redes de transporte de interés comunitario. En 1994 fueron ya avanzadas las propuestas de red ferroviaria de alta velocidad, red de carreteras de gran capacidad y red de transporte combinado. Se ha tratado siempre de planteamientos flexibles y de carácter indicativo en el intento de definir los escenarios de futuro de las grandes infraestructuras de interés comunitario, contando para ello con las propuestas de los estados miembros. De esta forma, al identificar los proyectos prioritarios, se orientan también, o se fijan, los procesos de tomas de decisión nacionales, dado que los sistemas comunitarios de ayudas y de financiación incitan a incorporar el interés comunitario.

Por otro lado, la política común de transportes viene persiguiendo también, entre otros objetivos en materia ferroviaria, la desaparición del efecto frontera que aún existe debido a problemas infraestructurales, tecnológicos y de gestión de los operadores, con las directivas en materia de interoperabilidad ferroviaria como marco legal tendente a ello.

\section{3.a. La Red Europea de Ferrocarriles de Gran Velocidad.}

La gran velocidad ferroviaria, al mismo tiempo que se considera oficialmente un potente elemento articulador de una Red Transeuropea de Transportes, serviría también como elemento de ruptura del efecto frontera.

Incluso desde antes de que se decidiera implantar el ferrocarril de gran velocidad en España, se ha intentado desde diversas instancias -como la Unión Internacional de Ferrocarriles- relacionar la implantación del nuevo sistema ferroviario con la integración europea; será de esta suerte el medio que acercará a las poblaciones europeas entre sí. Pero el concepto de red europea trae consigo el polémico asunto del diferente ancho de vía en la Península Ibérica y el resto de Europa occidental.

Diríase que, de forma similar a como en los años setenta se planteó la relación directa entre las autopistas y el desarrollo regional, desde al menos los últimos quince años se pretende engarzar la gran velocidad ferroviaria con los ejes estratégicos europeos y con la integración de las regiones periféricas en el ámbito europeo más dinámico. Incluso se ha llegado a exageraciones o dislates tales

como identificar la red europea de ferrocarriles de gran velocidad con el "metro 
europeo".

La Comisión Europea sostiene que esta red de gran velocidad, en el contexto de la multimodalidad de las redes transeuropeas, constituirá necesariamente una acción considerable y eficaz para lograr la integración europea en el largo plazo. Así, desde 1984 la Comisión ha presentado diversas propuestas acerca del enlace de las grandes ciudades europeas mediante líneas ferroviarias de gran velocidad, nuevas o acondicionadas. Ya en 1986 propuso al Consejo de Ministros de Transportes la creación de una red comunitaria, contando siempre con el apoyo del Parlamento Europeo. El Esquema Director de la red europea de gran velocidad, propuesto en 1990 y posteriormente definido por el Grupo de Trabajo de Alto Nivel, tipificó las líneas férreas de interés comunitario según velocidades-tipo: líneas nuevas de gran velocidad, líneas adaptadas a $200 \mathrm{~km} / \mathrm{h}$, líneas mejoradas a $160 \mathrm{~km} / \mathrm{h}$ y líneas de conexión y prolongación. En lo que respecta a España, forma parte de esta red europea en el horizonte 2010 tanto el corredor Madrid-Vitoria-Dax como el Madrid-Barcelona-Perpiñán, dos proyectos ferroviarios de gran velocidad que fueron considerados prioritarios en la Cumbre de Corfú y en el Consejo Europeo de Essen, de 1994. Y, desde que el acuerdo bilateral España-Portugal de 2003 fijara las nuevas líneas internacionales de gran velocidad, deben sumarse también los itinerarios Lisboa-Madrid por Cáceres y Lisboa-Oporto-Vigo.

Por último, el Libro Blanco sobre la Política Europea de Transportes de 2001 ha planteado la actualización de la llamada lista de Essen, proponiendo la construcción de un nuevo acceso ferroviario de gran capacidad a través de los Pirineos (Zaragoza-Pau), la interoperabilidad de la red ibérica de gran velocidad y otros grandes proyectos transeuropeos.

\section{3.b. La Red Europea de Transporte Combinado}

La congestión de las redes de carreteras y los problemas de contaminación y gasto energético que derivan del transporte viario han inducido a plantear la potenciación del transporte combinado y la planificación multimodal. El Libro Blanco sobre el desarrollo futuro de la Política Común de Transportes (COMISIÓN EUROPEA, 1992) planteó las ideas de movilidad sostenible y de integración del sistema de transportes como objetivos fundamentales en la Política Común de Transportes. Con esta visión, la Comisión elaboró un Esquema director de líneas férreas sobre la base de los flujos de tráfico por carretera que podían ofrecer posibilidades al transporte ferrocarril-carretera, así como otro ti- 
po de transferencias de tráfico hacia la navegación interior. Con horizonte en el año 2005, este Esquema director proponía ampliaciones de capacidad de líneas, inversión en equipos y compatibilización de los diferentes sistemas ferroviarios (ancho de vía, gálibo, electrificación, reglamentaciones, etc.).

Fueron definidos una serie de corredores europeos de transporte combinado, como por ejemplo el eje Irún-Fuentes de Oñoro y su conexión con Madrid. Naturalmente, se pretende transferir tráficos de la carretera al ferrocarril en estos corredores seleccionados, favoreciendo la intermodalidad y las rupturas de carga en determinados puntos con potencial estratégico. Algo que aún está en mantillas en las políticas públicas de transporte en España, que parecen dejar al albur de la iniciativa privada la construcción de plataformas logísticas y centros intermodales.

En el Consejo Europeo de Essen (1994) se procedió a la aprobación de los 14 proyectos prioritarios europeos entre los 26 identificados por el "Grupo Christofersen", de forma que, junto a las líneas de gran velocidad, fueron incluidos proyectos de transporte intermodal y de carreteras de gran capacidad. Y en la cumbre de Dublín (1996), por otra parte, se acordó -a iniciativa portuguesatransformar el proyecto prioritario de autopista Valladolid-Lisboa en un proyecto de enlace multimodal basado en el transporte combinado ferrocarril-carretera y por tanto también en los centros intermodales. El ferrocarril del corredor Medina del Campo a Pampilhosa por la frontera de Fuentes de Oñoro y Vilar Formoso se vería, así, potenciado de algún modo aún sin precisar.

\section{LA PROBLEMÁTICA CUESTIÓN DEL ANCHO DE VÍA.}

El ancho de vía de la red ferroviaria de la actual Renfe se forjó principalmente en la segunda mitad del siglo XIX, cuando fue determinado por el Estado ateniéndose a un informe efectuado por tres insignes miembros del Cuerpo de Ingenieros (INFORME SUBERCASE, 1844). Fue una decisión estrictamente técnica, impecable sobre la base del conocimiento que entonces se tenía del nuevo medio de transporte, pero que apenas algunas décadas después quedó en entredicho debido a los avances tecnológicos del material motor. Sin embargo, cambiar el ancho significaba una fortísima inversión pública sin efectos significativos en la productividad. Por otro lado, no sólo todas las vías de la red de interés general del Estado español tenían este ancho de vía, sino además -casi obligadamente- las de Portugal, de forma que puede hablarse del ancho de vía ibérico. 
Para situar el problema del ancho de vía en los ferrocarriles, debe señalarse que no es correcto plantear el dilema del ancho de vía como una confrontación entre ancho europeo y ancho español. Tanto en la Europa occidental al norte de los Pirineos como en el Magreb y en otras regiones del mundo, existe el ancho de vía internacional o UIC (Unión Internacional de Ferrocarriles, 1’435 m), mientras que en otros muchos países los anchos son otros; en España y Portugal predomina un ancho de vía mayor (1'668 m) que bien podríamos llamar ibérico. Así, no debe obviarse que cualquier decisión sobre la cuestión del ancho afecta sobremanera a Portugal.

Desde comienzos del siglo XX, hubo varias propuestas oficiales de cara a modificar el ancho de vía de la red, generalmente en contra de la opinión del Ramo de Guerra, que advertía de la importancia estratégica de los ferrocarriles en la defensa nacional y de la sobrevenida ventaja de que el diferente ancho dificultara su uso logístico por parte de una ofensiva desde Francia.

La iniciativa más famosa fue la propuesta presentada en las Cortes para la construcción de un ferrocarril directo París-Algeciras por Madrid en vía de ancho internacional (Gaceta del 22 de enero de 1919). La Real Orden de 17 de enero de 1914 que había ordenado el estudio del anteproyecto para una nueva línea a explotar por el Estado no fijó los puntos de paso, ni siquiera el de la frontera francesa, sino que tan sólo condicionaba el proyecto como una comunicación directa y rápida, con vía doble e incluso tracción eléctrica. Levantó el proyecto una intensa polémica tanto de tipo técnico -qué clase de ferrocarrilcomo de tipo político -qué puntos de paso- que se manifestó sobremanera en la prensa periódica y en el Parlamento, hasta que todo quedó en una idea abandonada, sustituida por acortamientos en las líneas existentes, los llamados ferrocarriles "directos".

La planificación ferroviaria no volvió a plantear el asunto del diferente ancho de vía a no ser para desarrollar soluciones técnicas de cara al estrechamiento de los ejes de los trenes españoles para poder circular por Francia y otros países europeos. Una vez creada la Renfe en 1941, ni los planes de Reconstrucción de los años cincuenta ni los de Modernización de los años sesenta y setenta, ni el Plan General de Ferrocarriles de 1981 ni el Plan de Transporte Ferroviario de 1987 tuvieron en cuenta la eventual viabilidad o conveniencia de estrechar la red nacional. El gigantesco gasto que suponía cambiar de ancho de vía podía ser mejor empleado en modernizar la red. Además, el ancho no era la única "frontera técnica", sino también el tipo de electrificación, los sistemas y reglamentos 
de seguridad y circulación, etcétera.

Sin embargo, el Acuerdo del Consejo de Ministros del día 9 de diciembre de 1988 supuso un giro radical en esta cuestión. Como ha sido ya mencionado, se decidió construir dos nuevas líneas de gran velocidad, lo que no estaba previsto en el PTF de 1987, pero además se trataba de dos nuevas líneas de ancho internacional. En segundo término, se vinculó el ancho internacional a la construcción de futuras nuevas líneas y en tercer término se solicitó a Renfe un informe sobre la conveniencia, el modo y el coste de convertir la red de Renfe al ancho internacional.

Se abrió de esta manera un intenso debate -complejo y limitado- sobre la introducción del ancho internacional en la Península Ibérica, que duró hasta los primeros años de la década de los noventa. Renfe redactó con cierto retraso el informe que le fue encargado, pero su carácter reservado evitó controversias y cerró el debate técnico. La compañía no discutió la conveniencia u oportunidad del cambio de ancho, sino la forma de asegurar su viabilidad técnica y económica. De esta forma, pareció aconsejable acometer el cambio según subredes y tipos de líneas, y por fases, de tal manera que -con el fin de no disparar el coste total- buena parte de la red secundaria podría eventualmente quedarse fuera del proceso. Aún así, el presupuesto estimado era muy elevado y se advirtió de los problemas de gestión de las infraestructuras en el obligadamente largo periodo de transformación de la red. A raíz de esto, podía entenderse que la pretensión de estrechar toda la red de Renfe era inasumible por su elevadísimo coste (rondando el billón de pesetas), y estrechar tan sólo algunas de las líneas supondría un sobrecoste de gestión y la ruptura del efecto de red, a pesar de las tecnologías de la rodadura desplazable (que entonces sólo se habían desarrollado para el material remolcado).

En 1993, el PDI asumió el Acuerdo de 1988 introduciendo sendas líneas de gran velocidad (Madrid-Sevilla y Madrid-Barcelona-Frontera francesa) más otras dos nuevas (Madrid-Albacete/Valencia y Zaragoza-Pamplona-País Vasco) y otras dos por decidir su corredor o su ancho de vía (Madrid-Lisboa y MadridValladolid). Pero el asunto del cambio de ancho de vía para el conjunto de la red fue soslayado, aunque mientras tanto se instalaban traviesas polivalentes (que permiten cualquiera de los dos anchos de vía mediante una rápida adaptación) en las vías principales, aprovechando su renovación o acondicionamiento para altas prestaciones. Se intentaba casar dos perspectivas contradictorias: el enlace ferroviario rápido con Europa mediante nuevas líneas de ancho interna- 
cional y la consideración de éstas como armazón de la red férrea, o sea como líneas troncales de una red que exigía numerosos intercambiadores de ancho interiores.

El PDI de 1993 parecía defender, aunque sólo formalmente, la existencia de una sola red o de unas redes ferroviarias perfectamente malladas. El efecto de red de las infraestructuras férreas debía lograrse mediante la instalación de toda una serie de intercambiadores de ancho que permitieran su interconexión, de forma que ambas redes tendrían tráficos de viajeros y de mercancías. De ahí que las nuevas líneas de gran velocidad construidas o proyectadas hasta entonces tuvieran unas pendientes inferiores a 15 milésimas por metro, que debían permitir el tráfico de trenes de mercancías de pequeña o mediana carga por eje, frente al caso francés, que admitía pendientes muy superiores dada su especialización en trenes de viajeros, de menor peso.

El PIT de 2000 ha optado por una "duplo-red", es decir, por la creación de una nueva red en ancho internacional (bien de nueva construcción, bien acondicionando líneas preexistentes) y el mantenimiento y redefinición de la red ordinaria en su ancho ibérico. Según el propio ministro de Fomento en su comparecencia ante la Comisión de Infraestructuras del Senado en septiembre de 2000, el "plan de alta velocidad" beneficia a las mercancías, porque los corredores actuales no tienen capacidad para hacer compatible el tráfico de viajeros y el de mercancías en un contexto futuro en el que se atiendan las expectativas de crecimiento del tráfico. Así, según el ministro, "las dos redes superpuestas son la única respuesta física a un problema insoluble sobre una única red", estando previstas excepciones en los "sitios donde no caben los dos corredores", es decir, se convierte un problema de anchos en un problema de incompatibilidad de tipos de trenes por su dispar velocidad.

El horizonte, por lo tanto, está en una doble red de algún modo interconectada: la red de gran velocidad (o alta velocidad) y la red ordinaria (o convencional). Ahora bien, y esto es un asunto ciertamente relevante, la primera con el ancho de vía internacional y la segunda con el ancho de vía ibérico. Dos anchos de vía que se consolidan y que son identificados con unos servicios determinados: el ancho internacional para el transporte rápido de viajeros y para las mercancías de alto valor añadido y escaso peso, y el ancho ibérico para las cercanías y las mercancías. Los tráficos regionales, por su parte, podrían efectuarse en ambas redes según el caso, siempre que se aseguren intercambiadores y se disponga de trenes completos interoperables y de rodadura desplazable, o sea, ap- 
tos para los dos sistemas y los dos anchos.

Dos grandes riesgos o problemas emanados de esta decisión pueden ser identificados. Por un lado, las grandes dificultades que entraña la constitución de dos redes independientes, puesto que hay numerosos lugares donde físicamente no caben juntas o donde deben compartir la plataforma ferroviaria, y por otro lado el gran problema de las conexiones, es decir, los intercambiadores de ancho de vía que palien el planteamiento de redes separativas especializadas. Pero el PIT no dispone los criterios generales para resolver uno ni otro asunto y nada escrito al respecto se ha encontrado, salvo la contestación oral del ministro al representante del grupo parlamentario mixto en la comparecencia ante el Senado, arriba mencionada y entrecomillada.

De hecho, el reciente Libro Blanco de la Comisión Europea "La política europea de transportes de cara al 2010: la hora de elegir", de 2001, recoge la necesidad de acometer este importante asunto. En el apartado dedicado a los grandes proyectos de infraestructuras (págs. 58-62 del texto francés) la Comisión Europea sostiene la necesidad de actualizar la lista de los 14 proyectos prioritarios establecida en Essen proponiendo, entre otras cosas, actuar en los ferrocarriles ibéricos para convertirlos al ancho internacional en el horizonte del 2020. Es decir, se reconoce un plazo de 20 años para una cuestión estratégica que el PIT 2000-2007 apenas plantea.

Además, el PIT dibuja la red de alta velocidad y ancho internacional para el escenario del año 2010, pero no hace lo mismo con la futura red de ancho ibérico. Todo lo más, se aprecia la red ordinaria actual como fondo gris en el mapa horizonte de la red de alta velocidad. Se trata, por lo tanto, de una "duplo-red" desigual: la nueva red de alta velocidad como apuesta de futuro para comunicar las capitales provinciales, ciudades y fronteras, y la red ordinaria como un resto.

\section{PERSPECTIVAS DE FUTURO.}

Entre el arranque en 1988 del primer proyecto AVE y la expresa decisión del Plan de Infraestructuras de 2000, que plantea la construcción de una red de gran velocidad ferroviaria en España conectada con la futura red transeuropea aunque separada de la red ordinaria, se está plasmando en la práctica una doble red férrea.

La "red de alta velocidad" servirá a todas las ciudades de la Península, en el afán de que el nuevo ferrocarril no beneficie exclusivamente a las mayores me- 
trópolis, si bien se trata de una disposición de las líneas que responde fundamentalmente a la demanda actual (Madrid es origen o destino de algo más de la mitad de los trenes interurbanos de Renfe) y prevista, lo que da lugar a una fuerte estructura radiocéntrica, ciertamente discutible. Por otro lado, es muy relevante el hecho de que el PIT -y los Estudios Informativos de los proyectos ferroviarios que de él derivan- consideren la conveniencia de trazar las nuevas líneas pasando por las ciudades intermedias, potenciando el sistema urbano existente, aunque es preciso señalar que son las villas y los centros comarcales de servicios los que no se verán servidos por el nuevo sistema: tal es el caso de Toro (Zamora), por ejemplo.

La red de alta velocidad necesitará de un periodo de implantación no inferior a quince años, lo cual generará muy diversos problemas de gestión al ferrocarril en general. Y la creación de dos redes separadas en función de su especialización de servicios, cuya impermeabilidad se fundamenta en su distinto ancho de vía, exige cuando menos planificar sus interconexiones mediante intercambiadores de ancho para los futuros trenes susceptibles de circular por ambas redes, así como programar los escenarios intermedios en la implantación de la "duplored", con el fin de que el efecto red se mantenga en niveles suficientes.

Se ha vinculado la modernidad del sistema de gran velocidad con el ancho de vía internacional -algo técnicamente insostenible-, y con la integración europea -aunque los tráficos no lo justifican de ningún modo-, algo que se aprecia con claridad cuando se usa la engañosa y propagandística expresión de "ancho europeo". Pero la introducción del ancho internacional en España, muy poco debatida en los círculos técnicos, lleva sobre sí el riesgo de generar unos problemas excesivos en relación con los beneficios, dado que las fronteras nacionales desaparecerán tal vez a costa de generar fronteras técnicas interiores que limitarían la eficacia de las inversiones.

\section{BIBLIOGRAFÍA.}

ARDUIN, Jean-Pierre (1991). «Las líneas de alta velocidad y el acondicionamiento del territorio» en la revista $O P \mathrm{n}^{\circ} 22$. Colegio de Ingenieros de Caminos de Cataluña, Barcelona.

ASSOCIATION DES VILLES TGV (1991). TGV et aménagement du territoire. Un enjeu majeur pour le développement local. Fruto de las actas del Primer Coloquio de la Asociación, celebrado en Creusot en octubre de 1990. Syros "Alternatives". París. 161 págs. 
AUPHAN, Étienne (1999). «Les gares TGV régionales: un exemple de contreaménagement du territoire» en Hommes et terres du Nord $\mathrm{n}^{\circ}$ 1, págs.14-20.

BAVOUX Jean Jacques \& CHARRIER, Jean Bernard (1994). Transports et structuration de l'espace dans l'Union européenne. Universidad de Borgoña. Masson. París. Sobre todo el capítulo 6 "Transports et villes", págs.111-119.

BAZIN, Jean-François (1988). Le TGV Atlantique. La seconde génération des trains à grande vitesse. Éditions Ouest-France. Rennes. 158 págs.

BIEBER, A. (1990). «Influence de la grande vitesse sur la restructuration de l'espace européen» en Recherche, transports, sécurité no 27. Págs.57-62.

BLIER, Gérard (1991, 1993, 1996). Nouvelle géographie ferroviaire de la France. Éditions La vie du rail. Volúmenes I ("Le réseau. Structure et fonctionament") de 1991, II ("L'organitation régionale du trafic") de 1993, y III ("L’impact du chemin de fer") de 1996. París.

BRUNET ESTARELLES, Pere \& SERVERA NicolaU, J. -coord.- (1992). I Seminario sobre el ferrocarril. Seminario celebrado en Palma en septiembre de 1991. Servicio de Publicaciones de la Universidad de las Islas Baleares. Palma de Mallorca. 143 págs.

BURMEISTER, Antje \& JOIGNAUX, Guy -dir.- (1995). Infrastructures de transport et territoires. Approches de quelques grands projects. Coloquio en Lille, INRETS y TRACES. Ed. L'Harmattan. París.

CANIAUX, Michel (1998). TGV: comment éviter un gâchis annoncé? L'Harmattan. 192 págs.

CENTRE EuropeEn Du DeVElopPEMENT Regional: Le défi régional de la grande vitesse. CEDRE, París. 205 págs.

CENTRO De ESTUdios URBANísticos, MUNICIPALES Y TERRITORIALES (1989). Monográfico «Tren de alta velocidad: oportunidad del debate» en CEUMT, la revista municipal $\mathrm{n}^{\circ} 111$, de sept-89. Barcelona. 69 págs.

ClAISE, Gérard \& DUCHIER, Didier (1993). Des observatoires d'effects TGV: réflexions méthodologiques. $6^{\circ}$ Entretiens sur Villes et TGV. Centre Jacques Cartier. Lyón.

Colegio De Ingenieros De CAMINos, CANALES y PUERTOS (1989). Jornadas sobre la introducción del ancho internacional en el ferrocarril español, celebradas en Madrid en abril de 1989. Ponencias. Madrid.

COLEGIO De InGENIEROS De CAMINOS, CANALES Y PUERTOS (1991 y 1992). Monográficos de la revista $O P$ : «el ferrocarril I» nº 22 (invierno 91), 107 páginas, «el ferrocarril II» ${ }^{\circ} 23$ (primavera 92), 111 páginas, y «el ferrocarril III» $\mathrm{n}^{\circ} 24$ (verano 92), 127 págs. Barcelona. 
COMISIÓN EUROPEA (1991). Libro Blanco sobre el futuro desarrollo de la política común de transportes.

COMISIÓN EUROPEA (1999). ETE, Estrategia Territorial Europea. Hacia un desarrollo equilibrado y sostenible del territorio de la Unión Europea. Estrategia acordada en la reunión informal de ministros responsables de ordenación del territorio en Postdam, mayo de 1999. Luxemburgo. 106 págs.

COMISIÓN EUROPEA (2001). Luz verde para Europa. Los proyectos de transporte financiados por el Fondo Europeo de Desarrollo Regional y el Fondo de Cohesión. Luxemburgo. 35 págs.

COMISIÓN EUROPEA (2001). Libro Blanco: la política europea de transportes de cara al 2010: la hora de elegir.

EMANGARD, Philippe (1990). "Du TGV Paris-Lyon au schéma directeur ferroviaire européen" en L'information géographique $\mathrm{n}^{\circ}$ 54. Págs.110-112.

ESTEVAN, Antonio \& SANZ ALDUÁN, Alfonso (1996). Hacia la reconversión ecológica del transporte en España. Comisiones Obreras, Bakeaz y Los Libros de la Catarata. Madrid.

FARIÑA ToJo, José, LAMíQuiz DAUdÉn, Francisco \& PoZUETA ECHAVARRI, Julio (2000). Efectos territoriales de la implantación de infraestructuras de accesos controlados, $\mathrm{n}^{\circ} 29$ de Cuadernos de Investigación Urbanística, ETSAM, Madrid. 95 págs.

FIDALGO, Pablo, PASTOR, Francisco \& ROMÁN, Manuel (1996): «Alta velocidad y Ferrocarril en España», 5 págs. en www.fundicot.org.

FOURNIAU, Jean Michel (1990). «De la ligne au réseau à grande vitesse. Le concept de réseau dans l'émergence des grandes vitesses en France» en Revue $d^{\prime}$ 'histoire des chemins de fer $\mathrm{n}^{\circ} 2$. Association pour l'histoire des chemins de fer en France. París. Págs.145-164.

FUNDACIÓN DE LOS FERROCARRILES ESPAÑOLES (1989). La introducción del ancho internacional en la red ferroviaria española. Fundación de los Ferrocarriles Españoles, Serie Documentos no 4. Madrid. 306 págs.

FUNDACIÓN DE LOS FERROCARRILES ESPAÑOLES (1993). Un año de alta velocidad. Del proyecto a la experiencia. Fundación de los Ferrocarriles Españoles, Serie Documentos $n^{\circ} 11$. Madrid. 454 págs.

GrouPEMENT Pour L'ETUde Des TRANSPORTS URBains Modernes -GetuM(1992a). Número especial «TGV: 1. La logique de la grande vitesse» en Transports urbains $\mathrm{n}^{\circ} 74$, enero-marzo de 1992. Courbevoie.

GROUPEMENT POUR L'ETUdE DES TRANSPORTS URBAINS MODERNES -GETUM(1992b). Número especial «TGV: 2. Aménagement du territoire» en Transports 
urbains $\mathrm{n}^{\circ} 75$, abril-junio de 1992. Courbevoie.

GrouPEMENT POUR L'ETUdE DES TRANSPORTS URBAINS MODERNES -GETUM(1996). Monográfico «Transports et territories dans le Nord-Pas de Calais», Transports urbains $\mathrm{n}^{\circ}$ 93, octubre-diciembre de 1996. Courbevoie.

HouÉE, Michel (1992). «Repercusión regional en Francia del tren de alta velocidad: evaluación de una experiencia», en el Seminario sobre Alta Velocidad ferroviaria y Desarrollo Regional: once años de experiencia europea en previsión y contraste de resultados. Madrid, diciembre de 1992.

MALO GAONA, José Antonio (1993). «Adopción de rampas máximas en alta velocidad» en la revista $O P \mathrm{n}^{\circ} 24$, Barcelona.

MATHIS, Philippe (1993). «L'effet paradoxal des transports à grande vitesse» en Destins atlantiques. Entre mémoire et mobilité. DATAR. Éditions de L'Aube, págs. 135-150.

MINISTERIO DE FOMENTO (2003). Plan de Infraestructuras del Transporte PIT 2000-2007. Madrid, 24 de octubre de 2003. En la página www.mfom.es. 101 págs.

Ministerio De ObRas PÚBliCAS Y TRANSPORTES (1993). Plan Director de Infraestructuras 1993-2007, Documento provisional, marzo-93. Secretaría General de Planificación y Concertación Territorial. Madrid. 379 págs.

Ministerio De OBRAS PÚBliCAS, TRANSPORTES Y MEDIO AMBIENTE (1994). Plan Director de Infraestructuras 1993-2007, $2^{a}$ edición, marzo-94. Madrid. 427 págs.

Ministerio De Obras Públicas, TRANSPortes Y Medio Ambiente [TAU PLANIFICACIÓN TERRITORIAL] (1993). Efectos urbanisticos y territoriales del tren de alta velocidad sobre las ciudades de Ciudad Real y Puertollano. Dirección General de Política Territorial y Urbanismo. MOPTMA, Madrid, octubre de 1993.

Ministerio De TRANSPORTES, TURISMO Y COMUNICACIONES (1987). PTF Plan de Transporte Ferroviario. Secretaría General Técnica, Centro de Publicaciones. Madrid. 45 págs.

NASH (1991). «Impacto socio-económico del tren de alta velocidad». Ciclo de conferencias sobre el transporte y las comunicaciones. Instituto de Estudios del Transporte y las Comunicaciones, Madrid, diciembre de 1991. MOPT.

NEBOT BELTRAN, Fernando (1991). «Velocidad punta y tiempo de recorrido», en la revista $O P \mathrm{n}^{\circ} 22$. Colegio de Ingenieros de Caminos de Cataluña. Barcelona. NEBOT BELTRAN, Fernando (1992). «Alta velocidad y planificación ferroviaria», en la revista $O P \mathrm{n}^{\circ}$ 23. Colegio de Ingenieros de Caminos de Cataluña. Barce-

Polígonos. Revista de Geografía, 11-12 (2001-2002); pp. 137-165. 
lona.

NÚÑEZ DEL CAMPO, Santos (1999): «España a dos velocidades», en www.cuerpo8.es/STOL/opinion/STOLdosvel.htm

OLLIVRO, Jean (1995). «Le réseau des lignes à grande vitesse: prégnance centralisatrice ou redéfinition de l'espace français?», págs. 196-219, en Revue d'histoire des chemins de fer $\mathrm{n}^{\circ} 12-13$, primavera-otoño-95. Association pour l'Histoire des Chemins de fer en France. París.

PIETRI, Jacques (1990). «Conséquences économiques et urbaines du TGV», comunicación en el tercer Congrès International de l'Association Mondiale des grandes métropoles, SEM de Massy.

PLASSARD, François (1988). «Le réseau TGV et les transformations de l'espace, la région Rhône-Alpes» en Les annales de la recherche urbaine $\mathrm{n}^{\circ} 39$.

PLASSARD, François (1991). «L'impact spatial des trains à grande vitesse en Europe» en el Seminario Transporte y Medio Ambiente, organizado por la Universidad Autónoma de Madrid, Miraflores de la Sierra, julio de 1991.

PLASSARD, François (1992). «L'impact territorial des réseaux à grande vitesse», págs. 243-262, en DERICKE, Pierre Henri -dir-: Espace et dinamiques territoriales. Économica. París. Págs. 243-262.

RouAULT, Dominique (1988). «L'effect TGV au Mans: trois fois plus de migrations quotidiens vers Paris» en Statistique et développement $\mathrm{n}^{\circ} 77$, de abril de 1988. INSEE-Pays de Loire. Págs. 11-14.

SANTOS Y GANGES, Luis \& CALVO ALONSO-CORTÉS, Santiago (1997). «El transporte combinado para el eje ferroviario Irún-Pampilhosa y su incidencia en la integración ibérica», comunicación en el II Congreso Ibérico de Urbanismo, ordenación territorial transfronteriza. AETU, Valladolid.

SANTOS Y GANGES, Luis (2001). «Ordenación del territorio y gran velocidad ferroviaria: el proyecto de la variante de Guadarrama», págs. 233-259, en Documentos del Instituto de Urbanística, $\mathrm{n}^{\circ} 5$. Instituto Universitario de Urbanística de la Universidad de Valladolid, Valladolid.

SANTOS Y GANGES, Luis (2002). El ferrocarril en la ciudad: estudio de las ciudades medias españolas. Tesis doctoral inédita. E.T.S. Arquitectura, Universidad de Valladolid. Valladolid. 823 págs.

SAVEL, Fréderique \& RABIN, Gilles (1992). La grande vitesse, outil d'aménagement du territoire? Association des Villes Européennes TGV. París. 89 págs.

SNCF (1987). Rapport de la Commission d'étude des tracés du T.G.V. Nord presidida por R. Rudeau. París, abril de 1987. 80 págs. 
SOCIETAT CATAlANA D' ORDENACIÓ DEL TERRITORI-SCOT- (1998). El TGV i les ciutats mitjanes catalanes. Quaderns de la SCOT n ${ }^{\circ} 8$, Institut d'Estudis Catalans. Barcelona. 104 págs.

TroIN, Jean François (1995). Rail et aménagement du territoire. Edisud, La Calade. 263 págs.

UNION INTERNACIONAL DE FERROCARRILES UIC: Grande vitesse, un réseau pour l'Europe. París 1992, reedición de 1993. 40 págs.

UNION INTERNACIONAL DE FERROCARRILES UIC: La grande vitesse en Europe, un nouvel élan. París 1994. 36 págs.

VARIOS AUTORES: Les aspects socio-économiques des trains à grande vitesse. Actas del seminario internacional homónimo, celebrado en París en noviembre de 1984. La Documentation Française. París 1985. 2 volúmenes de 240 y 702 págs.

WOLTERS, W. (1992). "Effets régionaux du transport à grande vitesse" en $L e$ défi regional de la grande vitesse. Région et Europe. Syros "Alternatives". Págs. 133-143.

\section{RESUMEN}

La introducción del ancho internacional en España para las nuevas líneas de gran velocidad ferroviaria ha desembocado en el planteamiento gubernamental de disponer de dos futuras redes férreas separativas en el horizonte del año 2010. La cuestión del ancho de vía se resolverá aparentemente optando por una "duplo-red" basada en la especialización y forzando la dualidad entre dos sistemas técnicos, dos velocidades, dos tipos de servicios, dos redes, dos anchos.

Palabras clave: Ferrocarril, línea de gran velocidad, planificación, red ferroviaria, ancho de vía, efecto de red, España.

\section{ABSTRACT}

The introduction of the international track gauge for the new railway high speed lines in Spain has lead to a governmental proposal consisting of having available two distinct railway networks by the year 2010 . The question of the track gauge will apparently be solved by opting for a double network based on the specialization, forcing a duality of technical systems involving two speeds, two kinds of services, two networks, two track gauges.

Keywords: Railway, high speed line, planification, railway network, track gauge, network effect, Spain.

\section{RÉSUMÉ}

L'introduction en Espagne de l'écartement international des voies pour les nouvelles lignes à grande vitesse ferroviaire a abouti à la proposition gouvernementale de disponer 
de deux futures réseaux ferroviaires distingués sur l'horizon de l'année 2010. Apparemment la solution pour la question de l'écartement des voies sera le choix d'un réseau doublé, s'appuyant sur la spécialisation et en forçant la dualité entre deux systèmes techniques, deux vitesses, deux types de services, deux réseaux, deux écartement des voies.

Mots clés: Chemin de fer, ligne à grande vitesse, planification, réseau ferroviaire, écartement des voies, effet de réseau, Espagne. 